\title{
The Material and Geometric Behaviors of a Composite Shell with a Quadratic Curve
}

\author{
Prof Kanggeun Park \\ Department of Architectural Engineering \\ I'ST Institute of Technology, Seoul, South Korea \\ Prof Mijin Park \\ Department of Architectural Engineering \\ Incheon National University, Incheon, South Korea
}

Byeongmok Park

ASM International, South Korea

\begin{abstract}
The objective of this study investigates the mechanical characteristics and nonlinear behaviors of the combined geometric and material nonlinear analysis for a quadratic composite shell. Structural composite materials are structurally bonded materials using laminated composites plates. Composite shells are widely used for structural components because they are light and strong, and have excellent corrosion resistance and high strength. They usually use carbon fiber, kevlar fiber or glass fiber. These composite members have anisotropic material whose properties are directionally dependent. The combined nonlinear behavior considering material and geometric nonlinear are analyzed for composite shells with a quadratic curve. The theoretical background and the analytical method for analyzing the behavior of these composite shells will be discussed and compared for the analytical results using NISA 2016 Software. From the results we can find new design concepts having more good structural functions by variously controlling physical properties. The material nonlinearity uses the elastoplastic hardening model, and the Full Newton-Raphson method is used in increment analysis.
\end{abstract}

Keywords - Mechanical Characteristics, Nonlinear Behavior, Composite Shells, Elastoplastic Hardening Model

\section{INTRODUCTION}

The curved shell members are widely used in various industrials and the curve has an effect on structural behaviors. The composite shell members are a combination of materials with different properties, and the physical properties are remarkably improved to satisfy the purpose and needs. The material consists of two or more materials which together produce desirable properties that cannot be achieved with any of the constituent alone. These composite shells are widely used for structural elements because they are light and strong, and they are excellent material properties for corrosion resistance, chemical resistance, electrical insulation and light weight. Such a composite material gives the capable of achieving a new design concept having excellent functions by variously controlling physical properties and flexibility of material performance. Composite shells are widely used in the aeronautical, aerospace, automotive, sports, architectural and civil engineering fields, and their applications are increasing in various industries in the future. It is becoming more and more important to analyze the mechanical properties of composite shells for specific conditions that cannot be achieved with conventional materials. Fiber reinforced composite materials consist of high strength and high modulus fibers in a matric material. In these composites, fibers are the principal load carrying members, and the matrix material keeps the fiber together, and protects fibers from being exposed to the environment. Fibers are stiffer and stronger than the same material in bulk form, whereas matrix materials have their usual bulk form properties. Fiber reinforced composite materials for structural applications are made in the lamina form of a thin layer. Structural members are formed by stacking the layers to achieve desired strength and stiffness. Fiber orientation in each lamina and stacking sequence of the layers can be chosen to achieve desired strength and stiffness for a specific application [1]. In this study, the behavior of geometric and material nonlinear synthesis of composite shells with the above physical properties is analyzed. The theoretical background and the analytical method for the analysis of these behaviors will be investigated. The present study will be entirely devoted to mechanics and analysis methods of a curved composite shell. 


\section{FAILURE CRITERION OF COMPOSITE MATERIALS FOR ANALYSIS}

\section{A. Mechanical Properties of Composite Materials-}

Composite materials are commonly formed in three different types which has classified with fibrous composites, particulate composites and laminated composites. The properties that can be improved by forming a composite material are stiffness, strength, weight reduction, corrosion resistance, thermal properties and fatigue life. A lamina is a typical sheet of composite material and represents a fundamental member block. A laminate is a collection of laminae stacked to achieve the desired stiffness and thickness. A fiber lamina consists of many fibers embedded in a matrix material, which can be a metal like aluminum or a nonmetal like thermoset or thermoplastic polymer. The fibers can be continuous or discontinuous, woven, unidirectional, bidirectional, or randomly distributed. Composite materials have high strength, high toughness, abrasion resistance, exhibit high reliability and stable performance in extreme environments [1]. Figure 1 is comparative stress-strain relationships of composite materials.

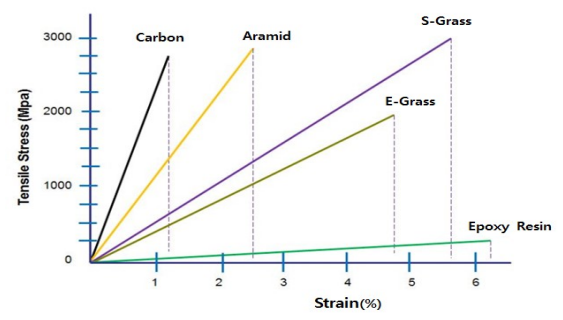

Figure 1. Comparative stress-strain relationships

Composite materials include particle-reinforced materials, fiber-reinforced materials, and structural composite materials. Generally, the particle strengthening material is isotropic, and the material has a geometry of dispersed phase in which the particles are continuous. Structural composites are structurally combined materials, which include laminated composites and sandwich plates. The reinforcing fibers of composite material are a load-bearing element, and a matrix is required to fix each of these fibers. Shear stress mainly affects the progress of fracture by combining the base material. The composite plate is generally anisotropic plate formed by stacking multiple orthogonal plates in order to improve the structural performance. Unlike isotropic plates, these anisotropic plates have different elastic moduli depending on the directions as shown in Figure 2. A composite material is called isotropic if its properties have the same value in all directions. The anisotropic material has three moduli of elasticity and three shear moduli. Fiber composite materials reinforced in uniaxial direction are transversely isotropic materials and are defined by two elastic moduli and one shear modulus. Materials such as woods are orthotropic materials defined by three elastic moduli [1].

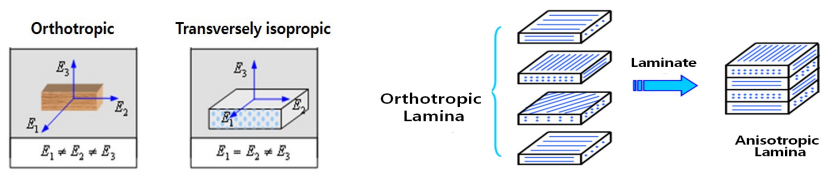

Figure 2. Orthotropic lamina and anisotropic lamina

\section{B. 3-D Composite Shell Element-}

The conventional finite element model is based on the 3-D theory. The 3-D laminate composite shell element in NISA 2016 software includes deformation due to membrane, bending, membrane-bending coupling and transverse shear effects, and is suited for modeling thick and thin laminated composite shells. The element consists of a number of layers of perfectly bonded orthotropic materials. The element has six degrees of freedom per node, but it possesses no rotational stiffness about the normal to the shell surface. The nonlinear analysis is solved by an iterative method. The Newton-Raphson iterative method is based on the tangent stiffness and the method has faster convergence for most applications.
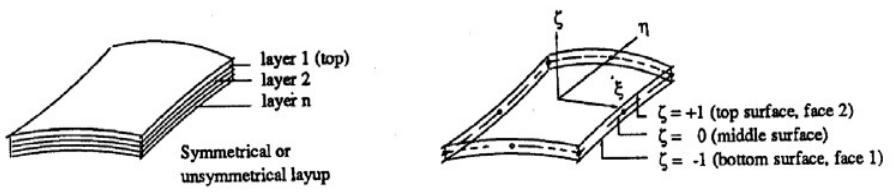

Figure 3. Layer setup and face numbering for top and bottom surface 


\section{Failure Criteria-}

Laminate failure occurs due to propagation of damage as the load is increased. To model this effect, a progressive failure approach is used in the nonlinear analysis. Each load step, Gauss point stress are used in the selected failure criterion. If failure occurred, a modification of the lamina properties was made at that point, which results in reduced stiffness. After nonlinear iterative displacement convergence is achieved, calculate stresses in the global coordinate at the middle of each layer. Transform the stresses to the principle material coordinates. If failure occurs, reduce the lamina moduli, compute laminate stiffness and restart the nonlinear analysis at the same load step. If no failure occurs, proceed to the next load step. Some research papers illustrate how both geometric and material nonlinearities influence the response of composite panels. Tsai[2,3] reported a survey of macroscopic failure criteria for composite material in journal of reinforced plastic and composite. Knight[5] studied factors influencing nonlinear static response prediction and test analysis correlation for composite panels in the journal of composite structures. The polynomial criteria are to describe the failure surface as a function of the material strengths. Generally, these expressions are based on the process of adjusting an expression to a curve obtained by experimental tests. The most general polynomial failure criterion for composite materials is Tensor Polynomial Criterion proposed by Tsai and $\mathrm{Wu}[2,3]$. This criterion is expressed in the following tensor notation.

$$
F_{i} \cdot \sigma_{i}+F_{i j} \cdot \sigma_{i} \cdot \sigma_{j}+F_{i j k} \cdot \sigma_{i} \cdot \sigma_{j} \cdot \sigma_{k} \geq 1
$$

The parameters $\mathrm{Fi}, \mathrm{Fij}$ and $\mathrm{Fijk}$ are related to the lamina strengths in the principal directions. For practical proposes, the third-order tensor Fijk is usually neglected. The general polynomial criterion reduces to the following general quadratic expression.

$$
F_{i} \cdot \sigma_{i}+F_{i j} \cdot \sigma_{i} \cdot \sigma_{j} \geq 1
$$

Considering that the failure of the material is insensitive to a change of sign in shear stresses, all terms containing a shear stress to first power must vanish: $F 4=F 5=F 6=0$. The explicit form of the general expression is the follows.

$$
\begin{aligned}
F_{1} \sigma_{1}+ & F_{2} \sigma_{2}+F_{3} \sigma_{3}+F_{4} \sigma_{4}+F_{5} \sigma_{5}+F_{6} \sigma_{6} \\
+ & F_{11} \sigma_{1}^{2}+F_{22} \sigma_{2}^{2}+F_{33} \sigma_{3}^{2}+F_{44} \sigma_{4}^{2}+F_{55} \sigma_{5}^{2}+F_{66} \sigma_{6}^{2} \\
& +2 F_{12} \sigma_{1} \sigma_{2}+2 F_{13} \sigma_{1} \sigma_{3}+2 F_{23} \sigma_{2} \sigma_{3} \leq 1
\end{aligned}
$$

The well-known quadratic failure criteria include those proposed by Tsai-Hill [3]. These quadratic criteria can be represented in terms of the general Tsai-Wu quadratic criterion varying the parameters $\mathrm{Fi}$ and $\mathrm{Fij}$ in order to ensure a good fit of the failure surface to the experimental results.

\section{Anisotropic elastoplastic model-}

Anisotropic elastoplastic material models are elastic perfectly plastic model and the elastic work hardening model. The elastic hardening model has a linear hardening model and piecewise hardening model. The yield criterion determines the stress level or the stress intensity level at the onset of plastic deformations. There are Hill's anisotropic yield criterions.

$$
\begin{aligned}
2 f\left(\sigma_{i j}\right)= & F\left(\sigma_{22}-\sigma_{33}\right)^{2}+G\left(\sigma_{33}-\sigma_{11}\right)^{2}+H\left(\sigma_{11}-\sigma_{22}\right)^{2} \\
& 2 L \sigma_{23}^{2}+2 M \sigma_{23}^{2}+2 N \sigma_{12}^{2}=1
\end{aligned}
$$

Where F, G, H, L, M and $\mathrm{N}$ are parameters of anisotropy, and 1, 2 and 3 are the three material principal directions. Linear terms are not included and therefore, no account for Bauschinger is possible. Modified Hill's criterion is as follows.

$$
3 f(\sigma)=M_{i j} \sigma_{i} \sigma_{j}-L_{i} \sigma_{i}-K=0
$$

Where $\mathrm{Mij}$, Li represent the variation of the yield stress with orientation as well as strength differentials between tensile and compression direction, $\mathrm{K}$ is a hardening parameter relating to the effective size of the yield surface. Linear terms are included in his equation, and therefore, Bauschinger effect can be accounted for.

\section{The Combined Material AND GeOMETRIC Behaviors of a QuAdRATIC COMPOSITE SHELL}

The highlight of this study is to obtain the load deflection curves at a central point load by the nonlinear static analysis considering the combined material and geometric nonlinearity using NISA 2016 software and compare with the results. A quadratic clamped shell is subjected to a central point load. The geometry is shown in Figure 4. The element type for analysis is 3-D eight node laminate composite shell element. In the principal material direction the material properties are defined in each Figure for elastic modulus, initial yield stress in material direction, initial yield stress in shear and work hardening parameters. The shell is modeled by 6 × 6 ( 36 composite shell elements). 
Symmetric boundary conditions are applied along the lines of the outer edges. Seven equal layers are taken through the thickness. The analytical results of the combined material and geometric analysis are Figure 5, 6, 7 and 8.
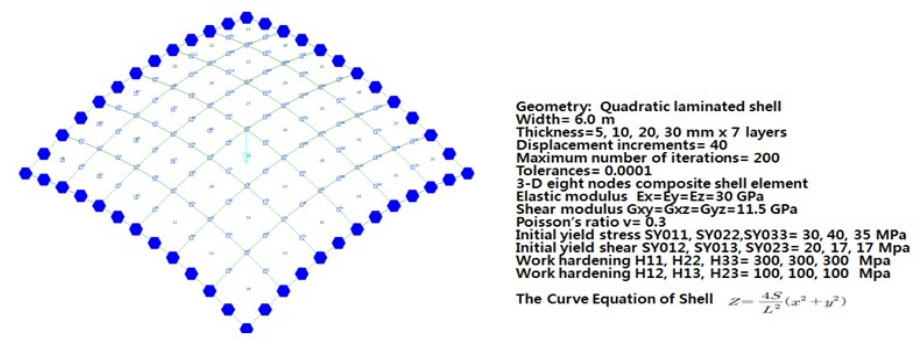

Figure 4. Geometry and modeling of a quadratic composite shell using 3-D composite shell element

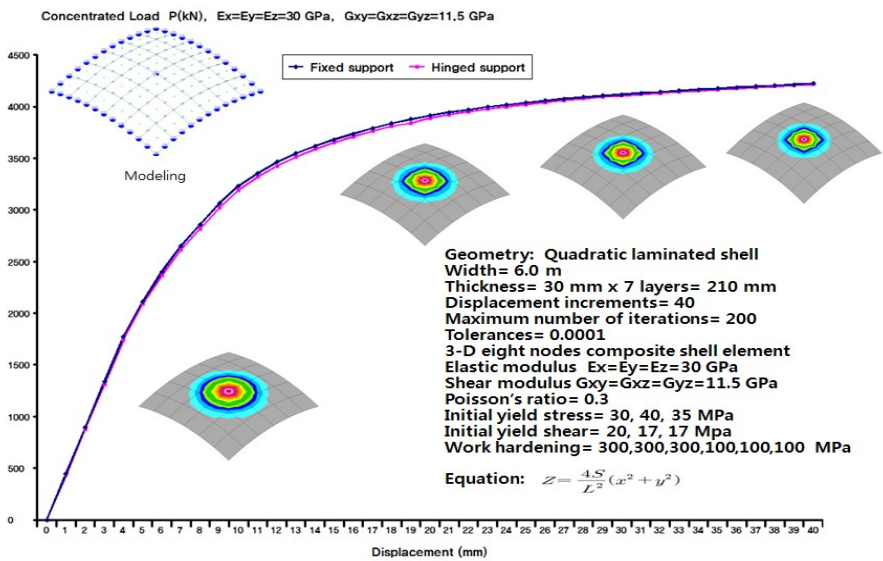

Figure 5. Load deflection curves of a quadratic shell for a central point load (Thickness: $30 \mathrm{~mm} \times 7$ layers=210 $\mathrm{mm}$ )

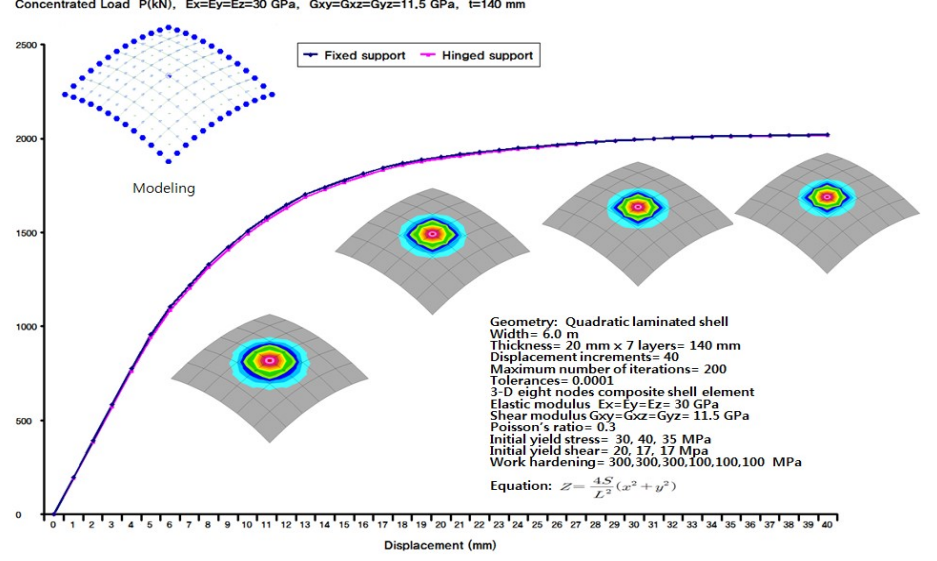

Figure 6. Load deflection curves of a quadratic shell for a central point load (Thickness: $20 \mathrm{~mm} \times 7$ layers=140 $\mathrm{mm}$ ) 


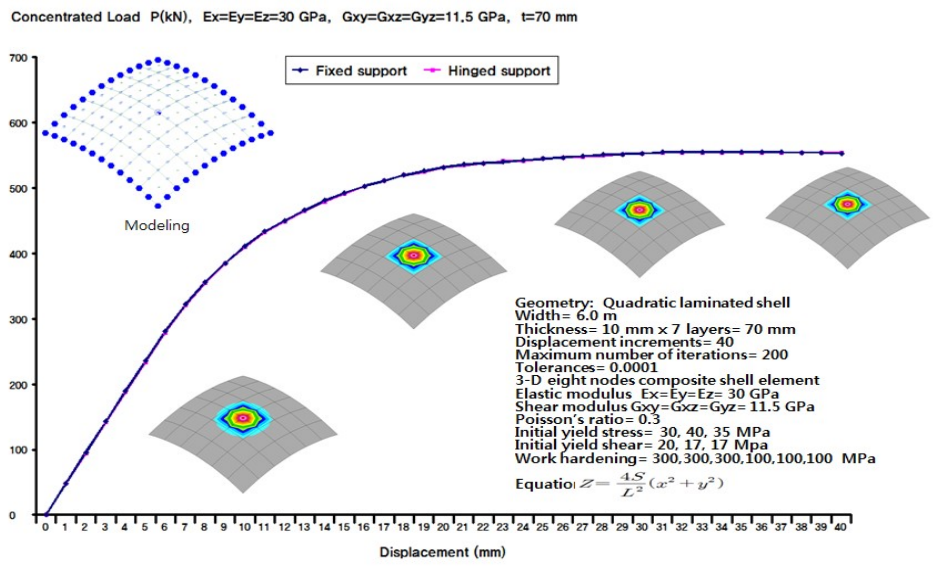

Figure 7. Load deflection curves of a quadratic shell for a central point load (Thickness: $10 \mathrm{~mm} \times 7$ layers=70 $\mathrm{mm}$ )

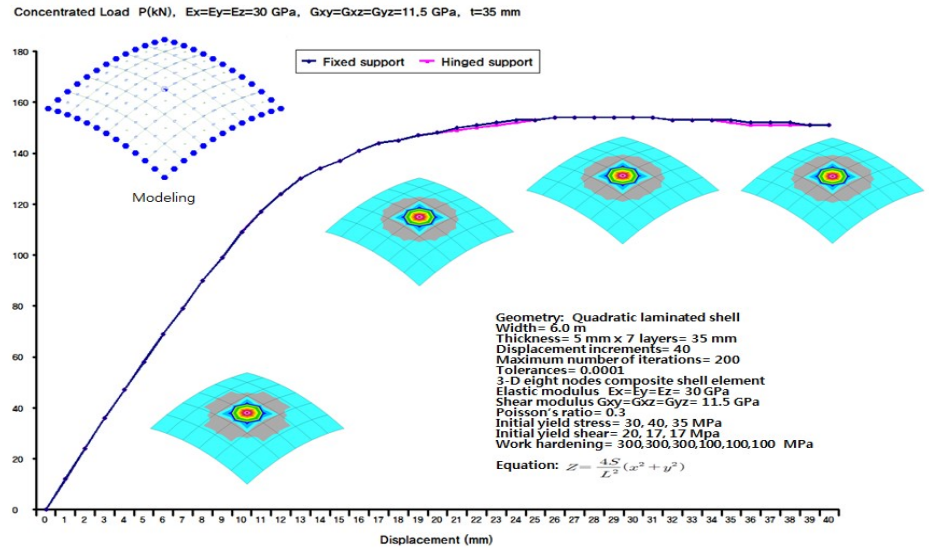

Figure 8. Load deflection curves of a quadratic shell for a central point load (Thickness: $5 \mathrm{~mm}$ x 7 layers $=35 \mathrm{~mm}$ )

\section{IV.CONCLUSION}

The objective of this research is to obtain the load deflection curves at a central point load by the nonlinear static analysis considering material and geometric nonlinearity.

(a)The analysis of anisotropic material should be considered to three moduli of elasticity and three shear moduli. Composite shells reinforced in uniaxial direction are transversely isotropic materials and can be defined by two elastic moduli and one shear modulus.

(b)The 3-D laminate composite shell element which includes deformations due to membrane, bending, membranebending coupling and transverse shear effects is suited for analyzing thick and thin laminated composite shells. (c)Anisotropic elastoplastic material model is possible to analysis by elastic perfectly plastic model and the elastic work hardening model. The elastic hardening model is possible to analyzing a linear hardening model or piecewise hardening model.

(d) The load deflection curves by fixed or hinged boundary conditions are plotted similarly.

(e)In load deflection curves of the nonlinear static analysis considering the combines material and geometric nonlinearity, good results are observed. The authors obtained useful information for the structural design of a quadratic shell from the estimation of structural capacity.

\section{ACKNOWLEDGEMENTS}

(a) This research was supported by a grant (17AUDP-B100343-03) from Architecture \& Urban Development Research Program funded by Ministry of Land, Infrastructure and Transport of Korean government.

(b) This research was supported by a grant (17CTAP-C115046-02) from Technology Advancement Research Program (TARP) funded by Ministry of Land, Infrastructure and Transport of Korean government 


\section{REFERENCES}

[1] J.N. Reddy, Mechanics of Laminated Composite Plates, CRC Press, 1997

[2] S.W. Tsai, E.M. Wu, "A General Theory of Strength for Anisotropic Materials," Journal of Composite Materials, 5, 58-80, 1971

[3] S.W. Tsai, "A Survey of maccroscopic failure Criteria for Composite Materials," Journal of reinforced Plastics and Composites, 3, 40-62, 1984

[4] Sun CT, Tao J. "Prediction of failure envelope and stress/strain behaviour of composite laminates," Composites Science and Technology $58,1125-36,1988$

[5] N.F. Knight, "Factors Influencing Nonlinear Static Response Prediction ans Test Analysis Correlation for Composite Panels," Composite Materials, 29, 13-25, 1994

[6] K.G. Park, D.W. Lee, "A Study on the Nature Inspiration Design of Eco-friendly Spatial Buildings," Journal of The Residential Environment Institute of Korea, 13(5), 1-20, 2016

[7] K.G. Park, D.W. Lee, D.I. Choe, "Mechanical Characteristics of Retractable Radial Cable Roof Systems," Journal of Korean Association for Spatial Structures, 17(2), 55-66, 2017

[8] K.G. Park, D.W. Lee, M.J. Park, "Mechanical Characteristics of Circular Cable Roof Systems," International Journal of Innovations in Engineering and Technology, Vol.8(3), 296-302, 2017

[9] K.G. Park, D.W Lee, D.I. Choe, M.J. Park, "Geometric Nonlinear Behaviors of Shallow Shells by Boundary Conditions," International Journal of of Latest Trends in Engineering and Technology, 8(4), 133-139, 2017 